\title{
Combined Endoscopic and Surgical Treatment of Severe Gastrointestinal Bleeding in a Patient with Heart Assist Device under Therapeutic Anticoagulation
}

\author{
Edris Wedi ${ }^{1,2}$, Mohamed Bounnah ${ }^{3}$, Riccardo Meme0 ${ }^{4,5}$ and Carlo Jung ${ }^{1,2}$ \\ ${ }^{1}$ Department of Gastroenterology and Gastrointestinal Oncology, Göttingen University Hospital, Göttingen, Germany, ${ }^{2}$ Department of \\ Gastroenterology, Nouvel Hôpital Civil, University Hospitals, Strasbourg, France, ${ }^{3}$ Medicine Department, Exploration and Digestive Endoscopy \\ Unit, Public Hospital Establishment Mohamed Boudiaf, El Khroub, Algeria, ${ }^{4}$ Department of Surgery, Nouvel Hôpital Civil and IHU Strasbourg, \\ University Hospitals, Strasbourg, France, ${ }^{5}$ Hepatobiliary Unit, Ospedale Generale Regionale F. Miulli, Acquaviva delle Fonti, Italy
}

Gastrointestinal (GI) bleeding is a common complication after heart assist device placement. Reasons for bleeding are multifactorial. Endoscopic therapy is the treatment of choice, whereas invasive procedures are avoided in these critically ill patients. We present the case of a 65-year-old male patient experiencing severe GI bleeding after left ventricular assist device (LVAD) and right ventricular assist device (RVAD) placement with therapeutic anticoagulation. Endoscopically, multiple gastric bleeding sources were found but could not be treated effectively due to a large blood clot. A combined endoscopic and surgical treatment was initiated, including gastrotomy for blood clot removal, surgical transgastric suturing, endoscopic over-the-scope clip (OTSC) placement and hemospray application. Postoperative endoscopic visualization showed effective bleeding control. The patient unfortunately died due to causes unrelated to the treatment. This case shows that a minimal invasive combination of endoscopic and surgical techniques can be an alternative treatment for severe upper GI bleeding in critically ill and anticoagulated patients. Clin Endosc 2017;50:598-601

Key Words: Heart assist devices; Gastrointestinal hemorrhage; Combined endoscopic and surgical treatment; Over-the-scope-clip; Hemospray

\section{INTRODUCTION}

In patients with left or right ventricular assist device (LVAD/RVAD), continuous therapeutic anticoagulation is essential to avoid clot formations. In general, these patients are often reported to have an increased risk of gastrointestinal (GI) bleeding up to $40 \% .^{1-3}$ The treatment of choice for upper GI bleeding is endoscopic therapy; drastic procedures, such as surgical gastrectomy, should be avoided wherever pos-

Received: January 20, 2017 Revised: March 16, 2017

Accepted: March 30, 2017

Correspondence: Edris Wedi

Department of Gastroenterology and Gastrointestinal Oncology, Göttingen University Hospital, Robert-Koch-Straße 40, Göttingen 37075, Germany

Tel: +49-1724060200, Fax: +49 (0) 551-39-669-2, E-mail: edriswedi@gmail.com

(cc) This is an Open Access article distributed under the terms of the Creative Commons Attribution Non-Commercial License (http://creativecommons.org/ licenses/by-nc/3.0) which permits unrestricted non-commercial use, distribution, and reproduction in any medium, provided the original work is properly cited. sible. Herein, we present the case of a multimorbid patient with LVAD/RVAD who needed continuous anticoagulation therapy. He was found to have a severe upper GI bleeding and was effectively treated with a combined multimodal endoscopy and surgical intervention to stop such bleeding. To our knowledge, this is the first case report describing this procedure.

\section{CASE REPORT}

A 65-year-old man with an ischemic cardiomyopathy due to coronary heart disease was admitted to our hospital. At admission, the physical examination revealed cardiogenic and septic shocks with laboratory results showing renal failure, metabolic acidosis (lactate, $52 \mathrm{mmol} / \mathrm{L}$ ), and anemia (hemoglobin, $9.6 \mathrm{~g} / \mathrm{dL}$ ). The patient had already received coronary stent placement and implantation of a defibrillator de- 


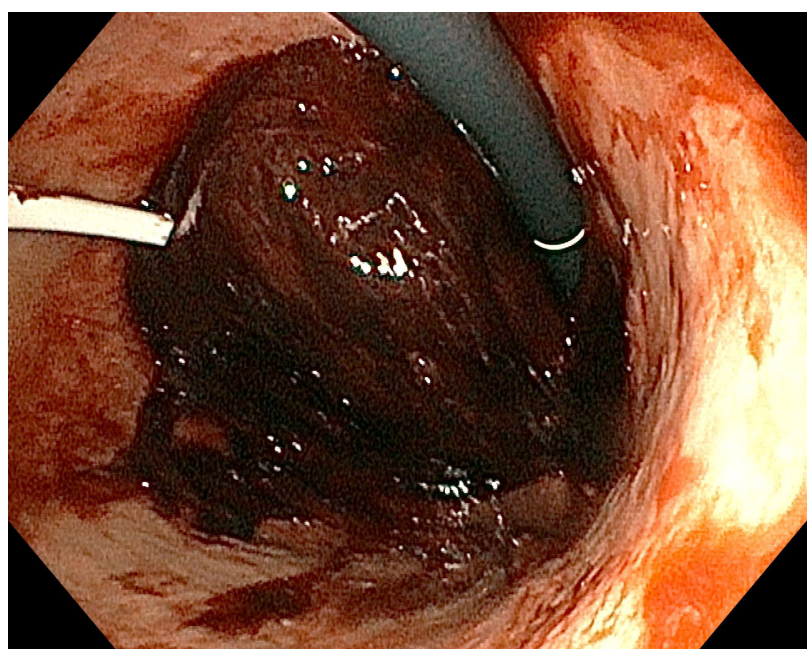

Fig. 1. $10 \mathrm{~cm}$ measuring bulky blood clot (retrovision).

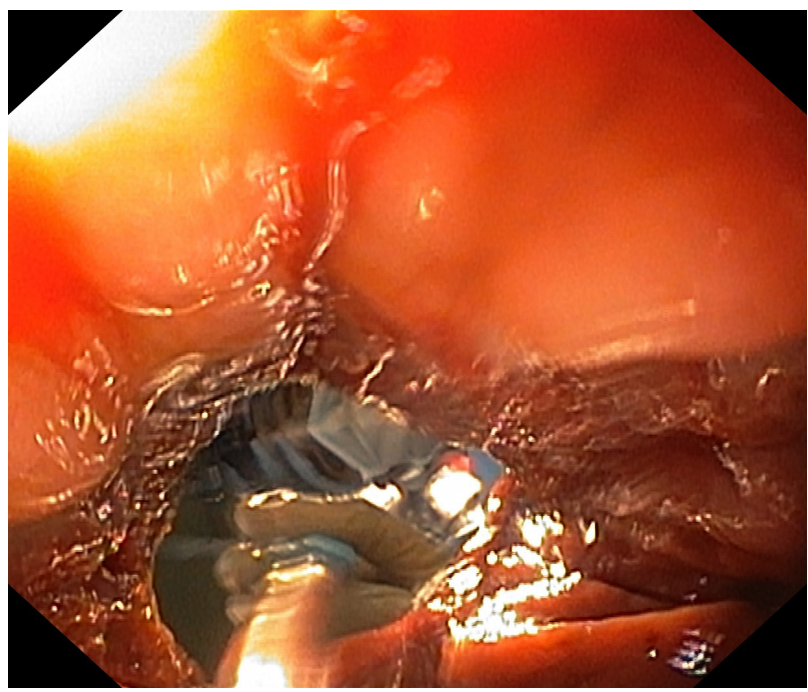

Fig. 2. Surgeons head seen from the gastroscope through an anterior gastrotomy.

vice due to severe congestive heart failure 2 months prior to this admission. Echocardiogram investigation demonstrated signs of severe left heart failure with a left ventricular ejection fraction rate of $25 \%$. Furthermore, the patient presented with a high level of catecholamines; therefore, the decision for LVAD/RVAD implantation was taken. Since then, the patient received an intravenous continuous heparin with a partial thromboplastin time goal of 60 seconds in addition to his antiplatelet therapy with aspirin. After his clinical symptoms improved, the RVAD could be removed on postoperative day (POD) 22. Twenty-four hours before RVAD removal, the patient presented with the first episode of hemoglobin level drop and melena. Emergency esophagogastroduodenoscopy (EGD) revealed traces of digested blood but no sign of active bleeding in the upper GI tract. Forty-eight hours later, the

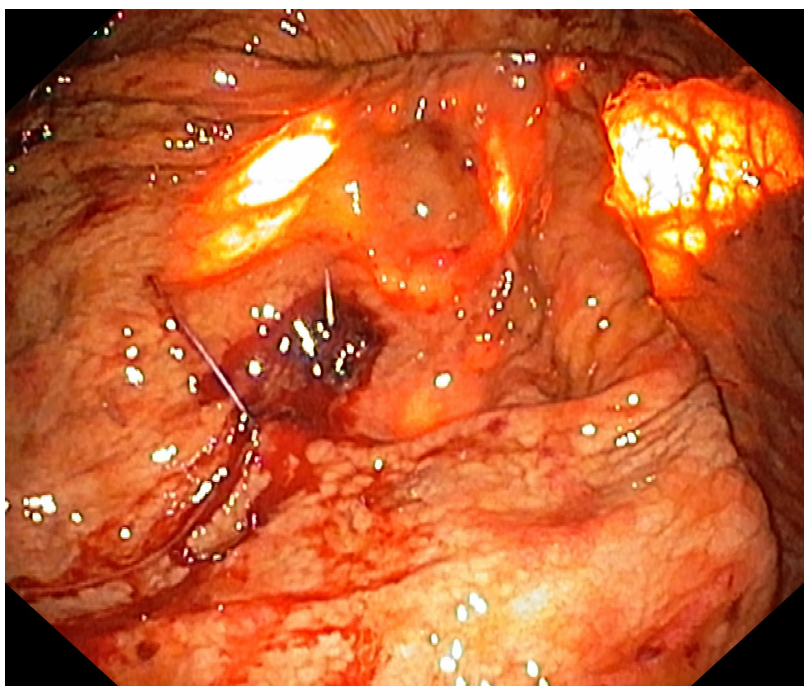

Fig. 3. Bleeding site (surgical suturing).

patient presented with hematemesis and melena. Repeated EGD showed a bulky blood clot (Fig. 1) measuring $10 \mathrm{~cm}$ in diameter but no active bleeding source. A full endoscopic evaluation was impossible at this point because of the blood clot. Owing to the large volume of the blood clot, an endoscopic extraction was also not possible; thus, after a discussion with our surgeons, a combined surgical and endoscopic therapy was decided upon. Angiography was also discussed as an option; however, as the risk of multiple bleeding sources owing to anticoagulation was high, we decided to use the combination of an endoscopic and surgical approach. The aim of this minimally invasive approach was to avoid performing a gastrectomy in this critically ill patient and to achieve a sufficient hemostasis using endoscopic techniques (over-the-scope clip [OTSC] placement and hemospray application).

First, the blood clot was removed by suctioning via mini-laparotomy and anterior gastrotomy by the surgeon (Fig. 2). Afterwards, the surgical gastric access was closed again, and the endoscopist was able to show the surgeon two actively bleeding vessels, which were located at the posterior wall of the greater curvature (Forrest Ia, Fig. 3) via diaphanoscopy.

The next step was the transparietal suturing of the two bleeding vessels performed by the surgeon, while the endoscopist visualized the bleeding site via diaphanoscopy to allow the surgeon to suture via the endoscopic image. From the luminal side, the endoscopist placed two 17.5 OTSCs (Ovesco Endoscopy AG, Tübingen, Germany) (type a) on each sutured vessel to ensure a firm hemostasis in this highrisk situation (Fig. 4). Further, the endoscopic examination revealed a 1.2-cm-large laceration in the fundus and two superficial vessels sitting on top of the fragile gastric mucosa; each was clipped with standard hemoclips. Additionally, the 


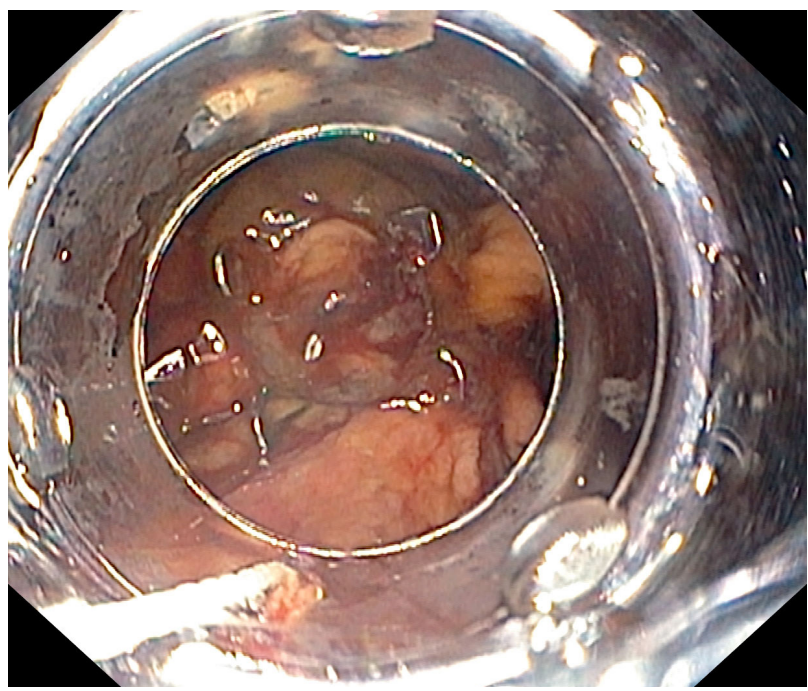

Fig. 4. Bleeding site (over-the-scope clip placement).

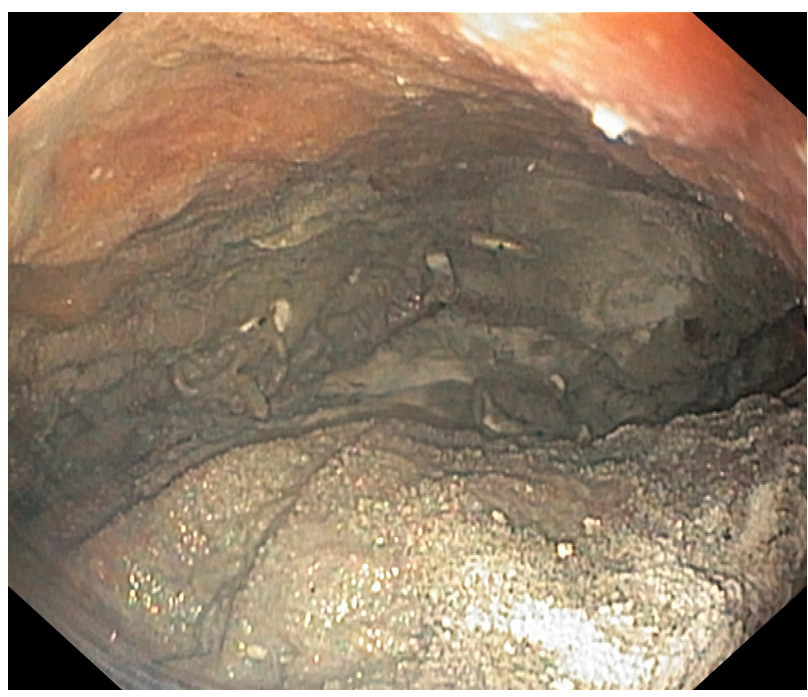

Fig. 5. Hemospray application.

patient presented with hemorrhagic gastritis; as such, endoscopy was finished by applying and covering all lesions with a hemospray (Cook Medical, Winston-Salem, NC, USA) (Fig. 5). Full anticoagulation was started 6 hours after the intervention. No other episodes of melena or hematemesis occurred. Endoscopic control 24 and $48 \mathrm{~h}$ after operation found all the clips in place and no further signs of active bleeding. Unfortunately, the patient presented with bronchial bleeding, hemodynamic shock, and neurological deterioration due to intracerebral hemorrhage on POD 7, resulting in his death.

\section{DISCUSSION}

LVADs have become a state-of-the-art therapy for ad- vanced cardiac heart failure. Nevertheless, these devices are associated with certain side effects, such as GI bleeding, owing to the need for therapeutic anticoagulation, acquired von Willebrand syndrome, platelet dysfunction, and increased incidence of arterio-venous malformations due to chronic low pulse pressure. ${ }^{1,4}$ The bleeding is predominantly located in the upper GI tract (48\%), and most of the lesions are peptic ulcers $(31.8 \%)$ or angiodysplasia $(29 \%)$. The incidence of GI bleeding in this population is reported to be as high as $18 \%-40 \%$ and therefore exceeds the risk of bleeding observed in patients who receive therapeutic anticoagulation for other medical indications. ${ }^{1,5-7}$ In a retrospective review, 101 LVAD patients were analyzed, and the event rate of GI bleeding was significantly higher in patients with non-pulsatile devices than in patients with pulsatile devices. ${ }^{3}$ An already presented possibility to reduce the risk of GI bleeding in patients with LVAD is to reduce the pump flow speed to increase the pulse pressure and reduce the anticoagulation therapy afterwards. ${ }^{89}$

The treatment recommendations for GI bleeding in patients with LVAD still need to be developed. Guidelines from major endoscopic societies do not yet exist for this special and complex indication.

In general, randomized controlled trials suggest adding proton pump inhibitors (PPIs) to antiplatelet therapy based on the results reporting a reduction of risk of GI bleeding by more than $60 \%$ in patients with average bleeding risks and by $89 \%$ in those already presenting a history of bleeding. ${ }^{10,11}$

However, the role of endoscopic treatment remains uncertain in these patients. Recently, a retrospective case study of 29 patients reported successful endoscopic treatment in nine patients with bleeding associated with LVAD. ${ }^{7}$ The treatment consisted of argon plasma coagulation, hemostatic clip placement, simple coagulation using a thermal device, and epinephrine injection. However, six of these patients undergoing endoscopic hemostatic therapy had rebleeding from the same location, despite PPI therapy.

Therefore, it is worth using new and combined techniques to halt bleeding such as that reported here. New endoscopic hemostatic techniques, such as OTSC placement or hemospray application, could improve patient care in this difficult and challenging clinical field. Further, combining endoscopic and surgical techniques could be an adequate therapy. The combination of OTSC placement and surgical suturing in the treatment of GI bleeding in patients with LVAD has not been reported yet.

In our case, endoscopic treatment alone was not feasible owing to the large blood clot that prohibited the visualization of the underlying bleeding vessels. A combined endoscopic and surgical approach seemed to be the safest and less inva- 
sive treatment. Otherwise, total gastrectomy or radiological embolization of an arterial vessel would have been the only likely alternative therapy. The combined strategy described here allowed the surgeons to visualize the origin of the bleeding and consequently to perform direct hemostatic suturing. At the same time, the surgical removal of the blood clot enabled the endoscopists to identify the multiple bleeding vessels. Following this, direct OTSC placement on the sutured vessels was feasible and effective. During the same gastroscopy, other small bleeding lesions were treated with standard hemoclips and hemospray. Hemospray was applied to maximize the therapeutic effects in this already multimorbid patient with hemorrhagic gastritis and minimize deleterious outcomes. As reported by Holster et al., hemospray application can achieve hemostasis even in a significant number of patients on antithrombotic therapy. ${ }^{12}$

We conclude that the presented case herein provides preliminary evidence that a combined approach of endoscopic and surgical treatment may be a good option in the treatment of severe bleeding or recurrent bleeding in LVAD/ RVAD patients. Such an approach is technically feasible, may reduce general surgical invasiveness in an already critically ill patient, and therefore, is likely to reduce the risk of postoperative complications. However, further studies are necessary to validate this method in larger patient cohorts, although it will certainly remain as the "last therapy option".

\section{Conflicts of Interest}

The authors have no financial conflicts of interest.

\section{REFERENCES}

1. Harvey L, Holley CT, John R. Gastrointestinal bleed after left ventricular assist device implantation: incidence, management, and prevention. Ann Cardiothorac Surg 2014;3:475-479.

2. Islam S, Cevik C, Madonna R, et al. Left ventricular assist devices and gastrointestinal bleeding: a narrative review of case reports and case series. Clin Cardiol 2013;36:190-200.

3. Crow S, John R, Boyle A, et al. Gastrointestinal bleeding rates in recipients of nonpulsatile and pulsatile left ventricular assist devices. J Thorac Cardiovasc Surg 2009;137:208-215.

4. Letsou GV, Shah N, Gregoric ID, Myers TJ, Delgado R, Frazier OH. Gastrointestinal bleeding from arteriovenous malformations in patients supported by the Jarvik 2000 axial-flow left ventricular assist device. J Heart Lung Transplant 2005;24:105-109.

5. Aggarwal A, Pant R, Kumar S, et al. Incidence and management of gastrointestinal bleeding with continuous flow assist devices. Ann Thorac Surg 2012;93:1534-1540.

6. Draper KV, Huang RJ, Gerson LB. GI bleeding in patients with continuous-flow left ventricular assist devices: a systematic review and meta-analysis. Gastrointest Endosc 2014;80:435-446.e1.

7. Kushnir VM, Sharma S, Ewald GA, et al. Evaluation of GI bleeding after implantation of left ventricular assist device. Gastrointest Endosc 2012;75:973-979.

8. John R. Current axial-flow devices--the HeartMate II and Jarvik 2000 left ventricular assist devices. Semin Thorac Cardiovasc Surg 2008;20:264-272.

9. John R, Kamdar F, Liao K, Colvin-Adams M, Boyle A, Joyce L. Improved survival and decreasing incidence of adverse events with the HeartMate II left ventricular assist device as bridge-to-transplant therapy. Ann Thorac Surg 2008;86:1227-1234; discussion 1234-1235.

10. Bhatt DL, Cryer BL, Contant CF, et al. Clopidogrel with or without omeprazole in coronary artery disease. N Engl J Med 2010;363:19091917.

11. Lai KC, Lam SK, Chu KM, et al. Lansoprazole for the prevention of recurrences of ulcer complications from long-term low-dose aspirin use. N Engl J Med 2002;346:2033-2038.

12. Holster IL, Kuipers EJ, Tjwa ET. Hemospray in the treatment of upper gastrointestinal hemorrhage in patients on antithrombotic therapy. Endoscopy 2013;45:63-66. 